04

\title{
Заполнение плоского щелевого объема тлеющим разрядом в поперечном магнитном поле и влияние магнитного поля на контракцию разряда
}

\author{
(C) М.С. Мокров, Ю.П. Райзер \\ Институт проблем механики им. А.Ю. Ишлинского РАН, \\ 119526 Москва, Россия \\ e-mail: mmokrov@gmail.com
}

(Поступило в Редакцию 18 октября 2017 г.)

Рассмотрен изучавшийся экспериментально тлеющий разряд постоянного тока в плоском щелевом объеме с электродами в плоскости щели и находящийся в поперечном току магнитном поле. Как и в эксперименте, разряд искусственно удерживается у одной из боковых диэлектрических границ объема, и под действием магнитного поля распространяется к противоположной диэлектрической границе, пока не стабилизируется. Двумерным расчетом нестационарного процесса продемонстрировано, что в магнитном поле разряд заполняет заметно больший объем (с меньшей плотностью тока на электродах), чем в отсутствие магнитного поля. Влияние магнитного поля проявляется также в том, что оно затрудняет контракцию разряда, существенно повышая предельный ток существования диффузного разряда. В приближении однородного вдоль тока положительного столба рассчитано, как контрагирует разряд вплоть до стационарного состояния. В расчете с магнитным полем при переходах из диффузного состояния в контрагированное и обратно проявился гистерезис.

DOI: 10.21883/JTF.2018.06.46012.2515

\section{Введение}

Поддержание слабоионизованной плазмы тлеющего разряда в большом объеме при не слишком низком давлении газа, желательное для ряда приложений, представляет известную трудность из-за свойственной разряду неустойчивости. В попытках решить эту общую проблему и подавить неустойчивость применительно к случаю $\mathrm{CO}_{2}$-лазерных систем в начале 90-х годов были предложены и экспериментально опробованы две схемы $[1,2]$, в которых ключевую роль играет использование внешнего магнитного поля. В экспериментах $[1,2]$, а также в [3], сделанных на установке [2], разряд зажигался в тонком плоском щелевом зазоре большой площади, принудительно удерживался у одной из боковых диэлектрических стенок зазора, где создавались более благоприятные условия для пробоя и ионизации, и помещался в поперечное току однородное магнитное поле $B=0.01-0.1$ Т. В экспериментах [1] использовались секционированные катод и анод. Для привязки разряда к краю разрядного промежутка крайние электроды располагались на меньшем расстоянии друг от друга, чем все остальные. Установка [1] использовалась как $\mathrm{CO}_{2}$-лазер, а работа [1] посвящена в основном оптическому устройству, и, кроме схематического рисунка установки не содержит никакой информации о разряде. Поэтому мы ориентируемся на установку и эксперименты [2,3], в которой для удержания основного разряда у левой границы щелевого зазора (рис. 1) использовался вспомогательный высокочастотный разряд небольшой мощности. Он мало влиял на основной процесс в стационарной стадии.
Благодаря действию пондеромоторной силы, пропорциональной $\mathbf{j} \times \mathbf{B}$, диффузный разряд расширялся вдоль электродов. Чем выше разрядный ток (текущий вдоль оси $x$ на рис. 1), тем больше ширина плоского разрядного слоя (вдоль оси $y$ ). При этом напряжение на разряде остается практически неизменным. Уменьшение магнитного поля приводит к уменьшению ширины разряда при фиксированном токе. При достаточно большом значении магнитного поля разряд заполняет весь объем камеры вплоть до противоположной (правой) диэлектрической стенки. Эксперименты также показали, что магнитное поле способствует существованию диффузного разряда, повышая пороговый ток перехода из диффузного состояния разряда в контрагированное.

Разряд в магнитном поле теоретически рассматривался в [3], где также были проведены дополнительные измерения с несколько отличными от [2] межэлектродным расстоянием и давлением газа. При теоретическом анализе проблемы в [3] разряд рассматривался в приближении квазинейтральной плазмы, бесконечной и однородной вдоль разрядного тока, так что все переменные зависели только от одной поперечной току координаты, обозначенной $y$ на рис. 1, и времени. Было выведено уравнение для плотности квазинейтральной плазмы, которое дополнялось уравнением теплопроводности для температуры газа. Численное решение уравнений при различных заданных значениях полного тока дало наблюдаемый эффект заполнения промежутка током в направлении вдоль электродов (у нас $y$ ) с вполне определенной плотностью тока, что авторы работы [3] назвали эффектом „нормальной плотности тока положительного столба“. Этот вопрос является проблематичным, потому 


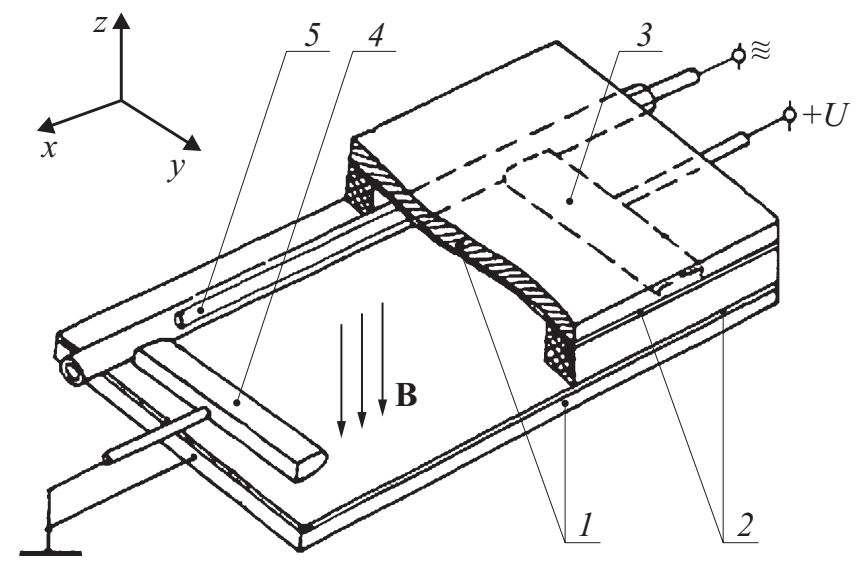

Рис. 1. Схема экспериментальной установки [2,3]. 1 - заземленные металлические пластины, образующие корпус разрядной камеры; 2 - тонкое диэлектрическое покрытие этих пластин, изолирующее разряд от пластин; 3 - анод, на которой подается постоянное напряжение; 4 - заземленный катод; 5 - вспомогательный ВЧ электрод, используемый для поджигания и удержания разряда у левой боковой границы разрядного объема. Система координат, принятая в нашем расчете, показана на рисунке отдельно. Однородное магнитное поле направлено вниз.

что в теории [3] вообще отсутствует катодный слой, в котором как раз и генерируется ток с нормальной плотностью. Это и послужило для нас одним из стимулов провести двумерный расчет заполнения промежутка разрядным током с учетом катодного слоя.

Вопрос о контракции разряда рассматривался в [3] в рамках стандартного линейного анализа на устойчивость по отношению к возмущениям, периодическим в пространстве. В численных расчетах [3] заполнения промежутка током контракция не проявилась, хотя нагрев газа, который обеспечивает ионизационно-тепловую неустойчивость разряда, был учтен. Результаты [3] не дают ответа на вопрос, как магнитное поле затрудняет развитие контракции. Это будет сделано ниже путем расчета нелинейной стадии развития контракции.

Настоящая работа имеет целью частично прояснить указанные выше процессы. Достаточно полную информацию о явлении дало бы численное моделирование разряда, как минимум двумерное, на основе решения системы уравнений непрерывности для плотностей электронов и ионов и уравнения электростатики для электрического поля с учетом нагрева газа. Реальную трехмерную геометрию щелевого зазора естественно свести к двумерной, просто путем учета ухода зарядов и тепла в направлении $z$, перпендикулярно плоскостям, ограничивающим узкий зазор.

Bce же даже в двумерной постановке, теоретический анализ проблемы сталкивается с трудностями по ряду причин. Одна из них связана со большими размерами устройства $[2,3]$ в направлениях $x$ и $y$, $L_{x} \times L_{y} \approx 22 \times 20 \mathrm{~cm}^{2}$ в [2] и $L_{x} \times L_{y} \approx 25 \times 14 \mathrm{~cm}^{2}$ в [3].
Большое межэлектродное расстояние $L_{x}$ для рассматриваемых давлений газа более чем на 2 порядка превышает толщину узкого катодного слоя, который необходимо разрешать на расчетной сетке. Поскольку размер устройства вдоль поперечного, „холловского“, направления $y$ сравним с продольным размером вдоль тока $x$, сильное сгущение сетки к катоду следует одновременно сопроводить сгущением сетки в области сильных градиентов величин на границе катодного пятна вдоль направления $y$. Положение этой границы заранее неизвестно, оно зависит от входных параметров задачи, прежде всего от тока. Такой расчет потребовал бы построения специальной, возможно, адаптивной сетки, применение которой представляет собой отдельную сложную задачу, выходящую за пределы данной работы. Другой проблемой, возникающей при анализе экспериментов [2,3], является то, что количественных данных измерений, с которыми можно было бы сравнивать теоретические результаты, в этих работах крайне мало.

Поэтому здесь мы ограничимся рассмотрением двух упрощенных задач.

(1) В рамках двумерной модели будет проведен более доступный расчет процесса заполнения разрядом промежутка, длина которого вдоль тока и ширина в несколько раз меньше реальных. Чтобы изучить, как зависят от магнитного поля параметры всего разряда, включая катодный слой, в этом расчете будем пренебрегать действием нагрева газа. Надо полагать, это действие проявляется в основном появлением контракции, которая здесь будет рассмотрена в одномерном приближении с учетом нагрева газа и с реальными экспериментальными размерами, и, как и в [3], в приближении квазинейтральной плазмы.

(2) Переход между диффузным и контрагированным состояниями в присутствии магнитного поля будет рассмотрен не путем линейного анализа, как в [3], а на основе нелинейных уравнений процесса вплоть до установления стационарных состояний.

В результате расчета второй задачи проявился эффект гистерезиса, причем весьма сильного. Скачкообразный переход от диффузного состояния к контрагированному при повышении тока происходит при токе, существенно превышающем ток обратного перехода, если ток уменьшать. Эффект выражен тем сильнее, чем больше магнитное поле.

Эксперименты [2,3] были выполнены для воздуха и $\mathrm{CO}_{2}$-лазерной смеси. Рассмотрение сложной плазмохимической кинетики в этих смесях увело бы нас далеко от основной цели работы - изучить возможность использования магнитного поля для создания диффузной плазмы для различных приложений (лазеры, плазмохимические реакторы и др.). Поскольку этот вопрос имеет общий характер, в качестве рабочего газа мы возьмем азот, ионизацию в котором описываем простейшей кинетической схемой, игнорируя процессы с участием колебательно- и электронно-возбужденных молекул. 
Рассматриваемая здесь двумерная задача о разряде в магнитном поле имеет много общего с решенной в [4-6], где рассчитывалось движение столба тлеющего разряда в плоской геометрии в поперечном току магнитном поле применительно к задачам аэрокосмической техники. Заданный в начальный момент посередине между боковыми границами столб разряда перемещался как целое в направлении действия силы $\mathbf{j} \times \mathbf{B}$. Когда он достигал боковой границы разрядного объема, счет прекращался. В отличие от нашего случая использованные в [4-6] граничные (и начальные) условия не допускали существования стационарного состояния разряда, на изучение характеристик которого как раз и направлена настоящая работа.

Отметим, что магнитное поле, как средство подавить контракцию, применялось и в других лазерных системах: на постоянном токе [7-10], на высокочастотном емкостном разряде [11]. В экспериментах $[7,8]$ разряд зажигался в трубке с диффузионным охлаждением, а $[9,10]$ в промежутке между двумя коаксиальными цилиндрами. В [7-10] использовалось поперечное току вращающееся магнитное поле, а эффект стабилизации разряда был связан с тем, что под действием силы $\mathbf{j} \times \mathbf{B}$ токовый столб все время перемещался в пространстве, так что газ локально не успевал сильно нагреться, тем самым распределение температуры газа в пространстве получалось более однородным, и неустойчивость подавлялась.

\section{Постановка задачи о двумерном численном моделировании и основные уравнения}

Будем рассматривать процесс протекания тока в системе, изображенной на рис. 1. Тлеющий разряд описываем уравнениями непрерывности для плотностей электронов $n_{e}$, ионов $n_{i}$ и уравнением Пуассона для $x$ и $y$ - компонент электрического поля $\mathbf{E}=-\nabla \varphi$, где $\varphi-$ потенциал. Уравнения записаны в системе координат, изображенной на рис. 1. Учтено, что проекция вектора магнитной индукции на ось $z$ отрицательна. В рамках рассматриваемой плоской двумерной геометрии производные всех величин вдоль $z$ исключаются и в правые части уравнений для электронов и ионов введены потери от амбиполярных потоков зарядов в плоские стенки камеры. Уравнения для электронов и ионов приводим без вывода. Общий их вывод можно найти в [4-6], а в упрощенном варианте - в [12]. Уравнения имеют вид

$$
\begin{gathered}
\frac{\partial n_{e}}{\partial t}+\frac{\partial \Gamma_{e x}}{\partial x}+\frac{\partial \Gamma_{e y}}{\partial y}=\alpha\left|\boldsymbol{\Gamma}_{e}\right|-b_{\mathrm{eff}} n_{e} n_{i}-v_{D} n_{e}, \\
\frac{\partial n_{i}}{\partial t}+\frac{\partial \Gamma_{i x}}{\partial x}+\frac{\partial \Gamma_{i y}}{\partial y}=\alpha\left|\boldsymbol{\Gamma}_{e}\right|-b_{\mathrm{eff}} n_{e} n_{i}-v_{D} n_{e} \\
\Gamma_{e x}=n_{e} u_{e x}=-n_{e}\left(\mu_{e \perp} E_{x}+\mu_{e \perp} \beta_{e} E_{y}\right) \\
-D_{e \perp} \frac{\partial n_{e}}{\partial x}-D_{e \perp} \beta_{e} \frac{\partial n_{e}}{\partial y}
\end{gathered}
$$

$$
\begin{aligned}
\Gamma_{e y}=n_{e} u_{e y}= & -n_{e}\left(-\mu_{e \perp} \beta_{e} E_{x}+\mu_{e \perp} E_{y}\right) \\
& -D_{e \perp} \beta_{e} \frac{\partial n_{e}}{\partial x}-D_{e \perp} \frac{\partial n_{e}}{\partial y}, \\
\mu_{e \perp}=\frac{\mu_{e}}{1+\beta_{e}^{2}}, \quad D_{e \perp}= & \frac{D_{e}}{1+\beta_{e}^{2}}, \quad \beta_{e}=\frac{\omega_{e}}{v_{e}}, \quad \omega_{e}=\frac{e B}{m_{e}}, \\
\Gamma_{i x}=n_{i} u_{i x}= & n_{i}\left(\mu_{i \perp} E_{x}-\mu_{i \perp} \beta_{i} E_{y}\right) \\
& -D_{i \perp} \frac{\partial n_{i}}{\partial x}+D_{i \perp} \beta_{i} \frac{\partial n_{i}}{\partial y}, \\
\Gamma_{i y}=n_{i} u_{i y}= & n_{i}\left(\mu_{i \perp} \beta_{i} E_{x}+\mu_{i \perp} E_{y}\right) \\
& -D_{i \perp} \beta_{i} \frac{\partial n_{i}}{\partial x}-D_{i \perp} \frac{\partial n_{i}}{\partial y}, \\
\mu_{i \perp}=\frac{D_{i}}{1+\beta_{i}^{2}}, \quad D_{i \perp}= & \frac{D_{i}}{1+\beta_{i}^{2}}, \quad \beta_{i}=\frac{\omega_{i}}{v_{i}}, \quad \omega_{i}=\frac{e B}{m_{i}}, \\
\Delta \phi= & -\frac{e}{\varepsilon_{0}}\left(n_{i}-n_{e}\right) .
\end{aligned}
$$

В (1), (2) $\boldsymbol{\Gamma}_{e}, \boldsymbol{\Gamma}_{i}, \mathbf{u}_{e}, \mathbf{u}_{i}-$ векторы плотностей потока и скоростей электронов и ионов в диффузионнодрейфовом приближении, $\mu_{e}, \mu_{i}, T_{e}, T_{i}, D_{e}=\mu_{e} T_{e}$ и $D_{i}=\mu_{i} T_{i}$ - электронная и ионная подвижности, температуры и коэффициенты диффузии, $\beta_{e}$ и $\beta_{i}$ - параметры Холла для электронов и ионов, $\omega_{e}, \omega_{i}-$ электронная и ионная циклотронные частоты, $v_{e}$ и $v_{i}$ - эффективные частоты столкновений электронов и ионов с нейтральным газом, $m_{e}$ и $m_{i}$ - массы электронов и ионов, $B$ - индукция магнитного поля. $\alpha-$ ионизационный коэффициент Таунсенда, $b_{\text {eff }}$ - коэффициент электронионной рекомбинации, $e-$ заряд электрона, $\varepsilon_{0}-$ диэлектрическая проницаемость вакуума.

В источнике $Q$ в правых частях (1), (2) считаем, что ионизационный коэффициент $\alpha$ зависит от магнитного поля как $\alpha=\alpha\left[E /\left(N \sqrt{1+\beta_{e}^{2}}\right)\right]$, где $N-$ плотность газа. Для аппроксимации $\alpha$ пользуемся формулой Таунсенда. Учет влияния магнитного поля на $\alpha$ уточняет ее величину по сравнению с принятой в [4-6], где влияние магнитного поля на скорость ионизации игнорировалось. Принятая нами зависимость $\alpha$ от магнитного поля может быть подкреплена следующим нестрогим рассуждением, основанным на элементарной теории [12]. Средняя энергия электрона $\varepsilon=m_{e} \bar{v}_{e}^{2} / 2$ при дрейфе в поперечном магнитном поле находится путем приравнивания скорости набора энергии, $e E^{2} \mu_{e} /\left(1+\beta_{e}^{2}\right)$, и скорости ее потерь, $v_{e} \delta \varepsilon$, где $v_{e}=\bar{v}_{e} / l, l$ - длина пробега, $\bar{v}_{e}=\left(8 k T_{e} / \pi m_{e}\right)^{1 / 2}-$ средняя тепловая скорость, $\mu_{e}=e /\left(m_{e} v_{e}\right), \delta-$ средняя доля энергии, теряемая электроном при столкновении с молекулой. Считая $l$ не зависящей от $\varepsilon[12]$, из выписанных соотношений находим $\varepsilon \approx e E l /\left(\sqrt{\delta} \sqrt{1+\beta_{e}^{2}}\right)$. Трактуя условно экспоненциальный множитель в таунсендовском коэффициенте как больцмановский фактор, содержащий среднюю энергию („температуру“) электронов, пропорциональную $E$, в аппроксимационную формулу Таунсенда для 
$\alpha$ в присутствии магнитного поля вместо $E$ подставляем $E / \sqrt{1+\beta_{e}^{2}}[13]$.

Слагаемое $v_{D} n_{e}$ в $Q$, где $v_{D}=D_{a} / \Lambda^{2}, 1 / \Lambda^{2}=(\pi / h)^{2}$, $h$ - толщина зазора, $D_{a}=\mu_{i} T_{e}-$ коэффициент амбиполярной диффузии плазмы с $n \approx n_{e} \approx n_{i}$, приближенно описывает уход зарядов в стенки щели за счет амбиполярной диффузии. Для простоты мы сохраняем этот член с $v_{D}$ и в областях приэлектродных слоев, хотя там диффузия заведомо не амбиполярна, - на результат это влияет мало.

Опыты [2,3] проводились в магнитных полях $B=$ $=0.01-0.1 \mathrm{~T}$. Даже для наибольшего $B$, $\omega_{i}=3.4 \times 10^{5} \mathrm{~s}^{-1}$, а при давлении газа $p=10$ Torr $v_{i}=$ $=4.7 \times 10^{7} \mathrm{~s}^{-1}$, так что $\beta_{i}=\omega_{i} / v_{i}=7 \times 10^{-3} \ll 1$, поэтому $1+\beta_{i}^{2}$ в (1) можно заменить единицей. Для электронов при тех же условиях $\beta_{e}=0.6$. Вне зависимости от величины $B$ ионная диффузия мала по сравнению с ионным дрейфом, поэтому слагаемые с $D_{i}$ в выражениях для потоков опускаем.

Граничные условия к системе (1)-(7) имеют следующий вид.

На аноде при $x=0, n_{i}=0 ; \varphi=U$, где $U-$ напряжение, приложенное к промежутку. Принимаем, что анод электроны не отражает [14]. В отсутствие магнитного поля этому соответствует граничное условие [14]

$$
\frac{n_{e} \bar{v}_{e}}{4}+\frac{1}{2} \Gamma_{e x}=0, \quad \Gamma_{e x} \approx-n_{e} \mu_{e \perp} E_{x}-D_{e \perp} \frac{\partial n_{e}}{\partial x} .
$$

Оно выведено там из кинетического уравнения для электронов в двучленном приближении при условии, что отсутствует односторонний поток электронов от анода в сторону положительного столба. Мы используем условие (8) в качестве граничного на аноде, несмотря на присутствие магнитного поля, считая, что магнитное мало влияет на движение электронов около самой поверхности анода. Заметим, что в обзоре [15] рассматривается кинетическое уравнение в двучленном приближении в присутствии магнитного поля в объеме однородной плазмы. Влияние магнитного поля на поведение электронов у поверхностей металлических электродов и диэлектриков там не рассматривается вообще. Этот вопрос представляет собой самостоятельную задачу.

На катоде при $x=L_{x}, \Gamma_{e x}=-\gamma \Gamma_{i x}$, где $\gamma-$ коэффициент вторичной ион-электронной эмиссии; $\varphi=0$. В выражении для $\Gamma_{e x}$, которое дается формулой (3), пренебрегаем последним слагаемым.

На левой боковой границе при $y=0$ граничное условие должно обеспечивать удержание плазмы от движения ее в сторону правой стенки. Для этого полагаем $\partial n_{e} / \partial y=\partial n_{i} / \partial y=0$. Для потенциала $\varphi$ условием служит равенство нулю полного тока $\Gamma_{y}=\Gamma_{i y}-\Gamma_{e y}=0$, где $\Gamma_{i y}$ и $\Gamma_{e y}$ даются формулами $(6),(4)$. Тем самым фактически задается значение поля $E_{y}=-\partial \varphi / \partial y$ на границе.

На правой боковой границе при $y=L_{y}$ отраженный от диэлектрической стенки электронный поток считаем отсутствующим. Для одностороннего потока принимаем формулу типа (8) с соответствующим знаком

$$
\frac{n_{e} \bar{v}_{e}}{4}-\frac{1}{2} \Gamma_{e y}=0
$$

где $\Gamma_{e y}$ дается формулой (4), в которой пренебрегаем слагаемым $\beta_{e} D_{e \perp} \partial n_{e} / \partial x$. Последнее возможно, поскольку в направлении оси $x$ при любых значениях у дрейфовый поток электронов много больше диффузионного, $\beta_{e} \mu_{e \perp} n_{e} / E_{x} \gg \beta_{e} D_{e \perp} \partial n_{e} / \partial x$. Для потенциала $\varphi$ условием при $y=L_{y}$, как и при $y=0$, служит равенство нулю полного тока $\Gamma_{y}=\Gamma_{i y}-\Gamma_{e y}=0$ через правую диэлектрическую границу.

Для вычисления падения напряжения на разрядном промежутке $U$ в систему уравнений (1)-(7) включалось уравнение внешней цепи. Поскольку в переходной стадии установления стационарного состояния ток в разрядном промежутке $I$ может сильно отличаться от тока во внешней цепи, в это уравнение включается слагаемое, описывающее накопление заряда $Q_{a}$ на электроде (аноде):

$$
\frac{d Q_{a}}{d t}=\frac{V_{0}-U}{R}-I, \quad Q_{a}=\varepsilon_{0} \int E_{a} d S,
$$

где $E_{a}$ - поле на аноде, $d S=d y \cdot h$ - элемент площади анода, $V_{0}$ - ЭДС источника питания, $R-$ внешнее сопротивление. Поскольку толщины электродов в $[2,3]$ не приведены, нам ничего не остается, как положить, что они совпадают с толщиной зазора $h$.

\section{Входные данные, методика двумерного расчета}

Для давления $p=10$ Torr как в эксперименте [2], толщина катодного слоя составляет $d_{c l} \approx 0.05 \mathrm{~cm}$, что в 400 раз меньше реального межэлектродного расстояния $L_{x}=22 \mathrm{~cm}$ и $L_{y}=20 \mathrm{~cm}$. Чтобы сократить объем вычислений, здесь мы ограничимся решением модельной задачи с существенно меньшими размерами $L_{x} \times L_{y} \approx 2 \times 3 \mathrm{~cm}^{2}$.

Расчеты сделаны для азота со следующим набором параметров. Ионная и электронная подвижности $\mu_{e} p=$ $=4.2 \times 10^{5} \mathrm{~cm}^{2}$ Torr $\mathrm{V}^{-1} \mathrm{~s}^{-1}, \quad$ и $\quad \mu_{i} p=1140 \mathrm{~V}^{-1} \mathrm{~s}^{-1}$, электронная температура $T_{e}=1 \mathrm{eV}, \quad D_{e}=\mu_{e} T_{e}=$ $=4.2 \times 10^{4} \mathrm{~cm}^{2} \mathrm{~s}^{-1}$,

ионной диффузией, не играющей роли, пренебрегаем $\left(D_{i}=0\right) ; \gamma=0.05$, толщина зазора $h=1 \mathrm{~cm}$. Формула для ионизационного коэффициента Таунсенда бралась из [12]

$$
\begin{aligned}
& \alpha=12 p \exp (-342 p / E), \quad E / p>100 \mathrm{~V} \mathrm{~cm}^{-1} \mathrm{Torr}^{-1}, \\
& \alpha=2.4 p \exp (-155 p / E), \quad E / p<100 \mathrm{~V} \mathrm{~cm}^{-1} \text { Torr }^{-1} .
\end{aligned}
$$

Система уравнений (1)-(7) интегрировалась численно методом установления до состояния, близкого к стационарному. Уравнения (1), (2), (7) приводились к конечно-разностному виду с помощью 
метода конечного объема на прямоугольной сетке с числом узлов $N_{x} \times N_{y}=100 \times 60$, которая сгущается к электродам. Входящие в уравнение (1) перекрестные члены $-\partial / \partial x\left[\beta_{e} /\left(1+\beta_{e}^{2}\right) D_{e} \partial n_{e} / \partial y\right]$ и $+\partial / \partial y\left[\beta_{e} /\left(1+\beta_{e}^{2}\right) D_{e} \partial n_{e} / \partial x\right]$ сокращаются, так как $\beta_{e}$ и $D_{e}$ не зависят от координат. Для электронного и ионного уравнений использовались неявные схемы „против потока“. Полученные в результате конечно-разностные аналоги уравнений (1), (2), (7) решались методом релаксации с прогонкой по линиям вдоль оси $x$. Для совместного решения уравнения (10) с уравнениями (1), (2), (7) использовался безытерационный алгоритм [16].

Начальное условие выбиралось так, чтобы разряд удерживался у левой границы камеры, как в экспериментах [2,3]. Это достигалось заданием однородных вдоль $x$ и слабонеоднородных вдоль $y$ распределений $n_{e}=n_{i} \sim 10^{6} \cdot \exp \left(-y / L_{y}\right) \mathrm{cm}^{-3}$ и однородного вдоль $x$ электрического поля. В первоначальной серии расчетов магнитное поле выключалось. Рассчитанные в результате распределения зарядов и поля при этом совпадали с теми, которые получаются в диффузионно-дрейфовом приближении $[5,6,12,17]$. Эти распределения служили в качестве начальных условий для вариантов с ненулевым магнитным полем.

Отметим, что в реальном эксперименте [2,3] локализация разряда у левой границы разрядной области достигалась путем зажигания в этом месте вспомогательного ВЧ разряда с помощью электрода, вставленного в щелевой зазор. ВЧ разряд горел в промежутке между диэлектрической трубкой, в которую был вставлен стержневой ВЧ электрод, и двумя заземленными металлическими пластинами, покрытыми диэлектриками и образующими щелевой зазор (см. схему на рис. 1). Для тлеющего разряда ВЧ разряд формально служит несамостоятельным источником внешней ионизации. Однако, поскольку мощность ВЧ источника в эксперименте [2] не превышала $50 \mathrm{~W}$, что почти на порядок меньше мощности разряда постоянного тока, ВЧ разряд не может конкурировать по скорости ионизации с разрядом постоянного тока. Поэтому, отказываясь от включения ВЧ разряда в модель, мы предполагаем, что он служит лишь в качестве начального источника затравочных зарядов на левом краю области для разряда постоянного тока. По мере развития ионизации в постоянном поле в балансе числа зарядов ВЧ разряд играет малую роль. Расчет комбинированного процесса с учетом ВЧ разряда сильно усложнил бы задачу и сделал ее трехмерной.

\section{Результаты двумерных расчетов}

Для иллюстрации эффекта заполнения разрядом плоского промежутка проделаны расчеты с различными значениями полного тока и магнитного поля. При фиксированном токе варьировалось магнитное поле, и, наоборот, в неизменном поле увеличивался ток. Разрядный ток регулируется выбором значений напряжения источника питания $V_{0}$ и внешнего сопротивления $R$.

Рис. 2 и 3 иллюстрируют влияние магнитного поля на разряд при двух значениях полного тока. Приведенные распределения всех величин соответствуют стационарному состоянию.

На рис. 2 рассмотрен случай достаточно малого тока, так что разряд без магнитного поля заполняет менее трети ширины промежутка $L_{y}$ (рис. 2,a). Как показывают расчеты, включение магнитного поля практически не влияет на полный разрядный ток и напряжение. Магнитное поле приводит к перераспределению тока и увеличению области, занимаемой разрядом в $y$-направлении (шириной разряда). Как видно из рис. 2, $c$, в поле $B=0.02 \mathrm{~T}$ разряд заполняет уже более половины ширины промежутка. При этом плотность тока на катоде при $B=0.02$ Т уменьшается почти вдвое по сравнению со случаем $B=0$, она падает от 12 до $6 \mathrm{~mA} / \mathrm{cm}^{2}$ (см. рис. 2, $d$ ). Тенденция к уменьшению с ростом $B$ наблюдается и в величинах плотности тока на аноде, плотностей зарядов в положительном столбе. Так, максимальная плотность зарядов в положительном столбе, которая в разряде без магнитного поля есть $n_{e} \approx n_{i} \approx 1.2 \cdot 10^{10} \mathrm{~cm}^{-3}$, в магнитном поле $B=0.01 \mathrm{~T}$ составляет $n_{e} \approx n_{i} \approx 8.2 \cdot 10^{9} \mathrm{~cm}^{-3}$, а в поле $B=0.02 \mathrm{~T}$ равна $n_{e} \approx n_{i} \approx 6.2 \cdot 10^{9} \mathrm{~cm}^{-3}$ (рис. $2, a-c$ ). Максимальные плотности тока на аноде при $B=0,0.01$ и 0.02 Т равны $j=16,7$, и $5 \mathrm{~mA} / \mathrm{cm}^{2}$ соответственно.

Если разрядный ток и магнитное поле достаточно велики, то разряд, расширяющийся в $y$-направлении, достигает правой стенки камеры. Этот случай показан на рис. 3. Без магнитного поля разряд занимает половину ширины камеры (рис. $3, a$ ). В магнитном поле $B=0.01 \mathrm{~T}$ разряд занимает три четверти расстояния до правой стенки (рис. $3, b)$, в поле $B=0.02$ Т в установившемся стационарном состоянии разряд достигает правой стенки (рис. 3,c). Как и в варианте с током $I=7.1 \mathrm{~mA}$, при включении магнитного поля максимальные значения плотности зарядов в положительном столбе и плотности тока на электродах снижаются (рис. 3, $a-d$ ). Плотности тока на катоде и аноде на протяжении значительной части площади электродов оказываются практически одинаковыми при всех рассчитанных значениях $B$, но от значений $B$ зависят (рис. $3, d$ ).

Рассчитанные распределения плотности тока для двух значений самого тока $I$ удобно привести на одном графике для каждого из трех принятых значений $B$, чтобы показать выполнение закона нормальной плотности тока. Это сделано на рис. 4.

Таким образом, процесс заполнения щелевого промежутка разрядом в магнитном поле для исследованного диапазона экспериментальных условий $[2,3]$ связан с расширением катодного пятна, плотность тока в котором уменьшается с ростом магнитного поля.

Тот факт, что в магнитном поле помимо основного тока, текущего вдоль оси $x$, ток имеет дополнительную $y$-составляющую, связанную с действием магнитного 

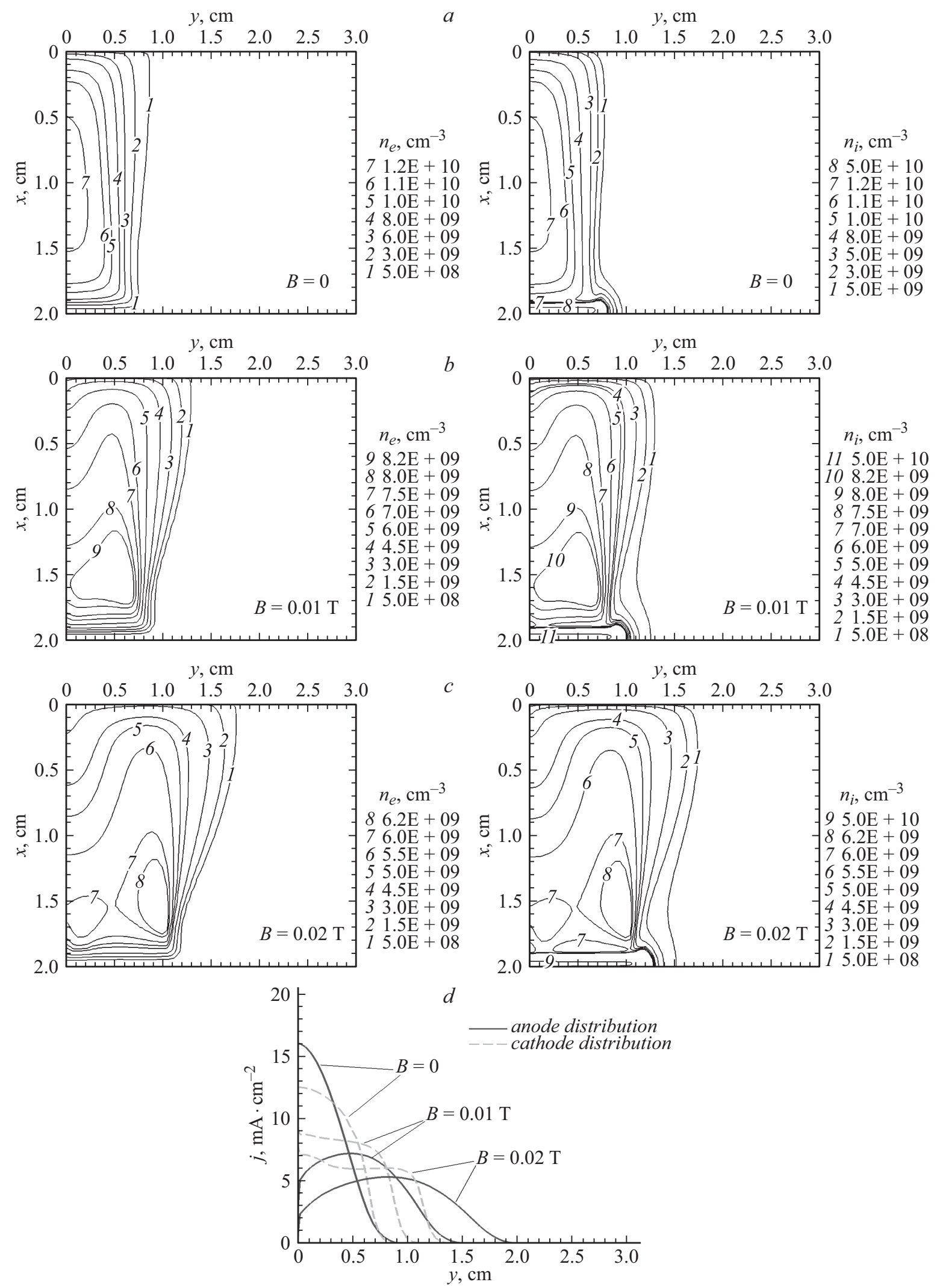

Рис. 2. Распределения плотностей электронов и ионов в различных магнитных полях, которые указаны на рисунках $a-c$; катод внизу, анод вверху. $a-$ плотности тока на катоде и аноде. Напряжение источника питания $V_{0}=2000 \mathrm{~V}$, внешнее сопротивление $R=2 \cdot 10^{5} \Omega$. Установившийся стационарный разрядный ток $I=7.1 \mathrm{~mA}$ и напряжение на разряде $U=574 \mathrm{~V}$ не зависят от значений магнитного поля $B$. 

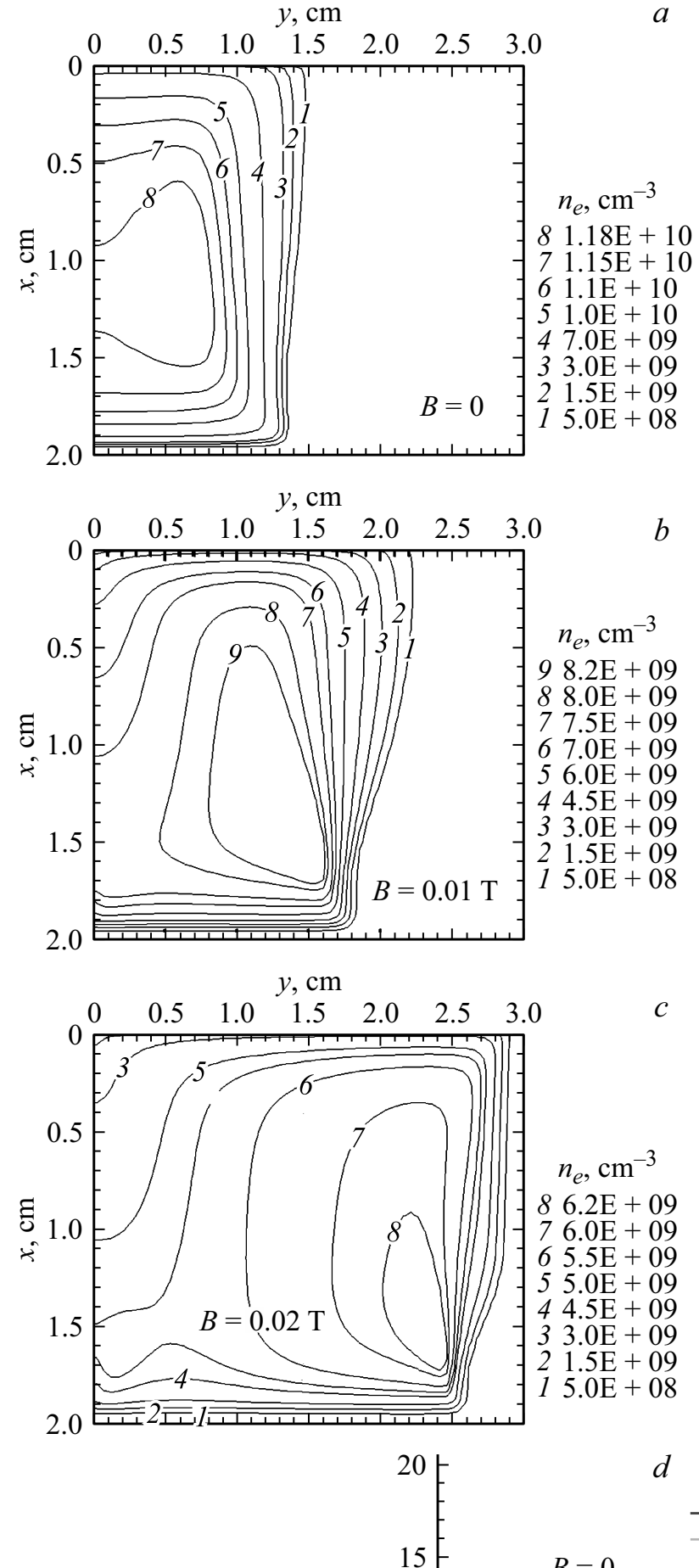

$d$
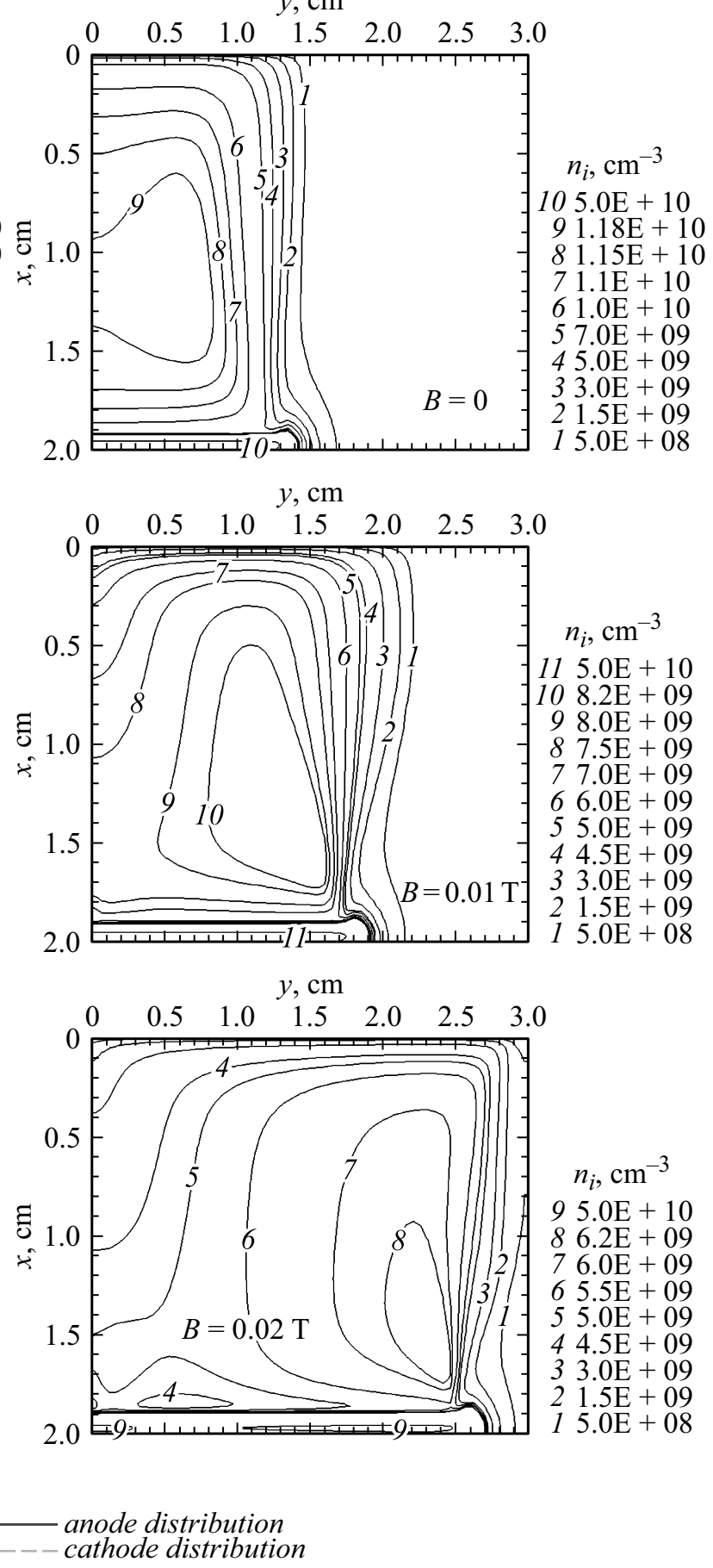

- anode distribution

$B=0.01 \mathrm{~T}$

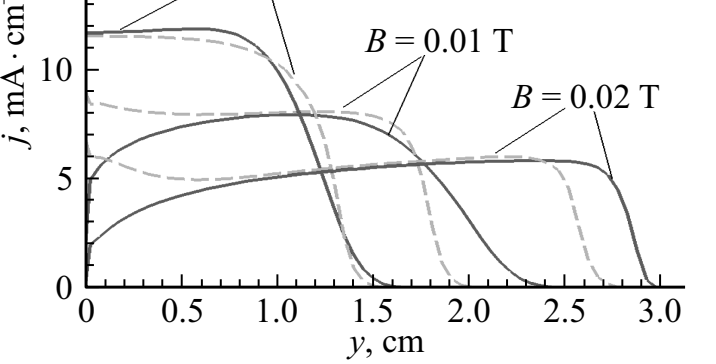

Рис. 3. То же, что и на рис. 2, но при другом внешнем сопротивлении, $R=1 \cdot 10^{5} \Omega$, при котором стационарный разрядный ток $I=14 \mathrm{~mA}$ и напряжение на разряде $U=574 \mathrm{~V}$ также независимо от магнитного поля. 

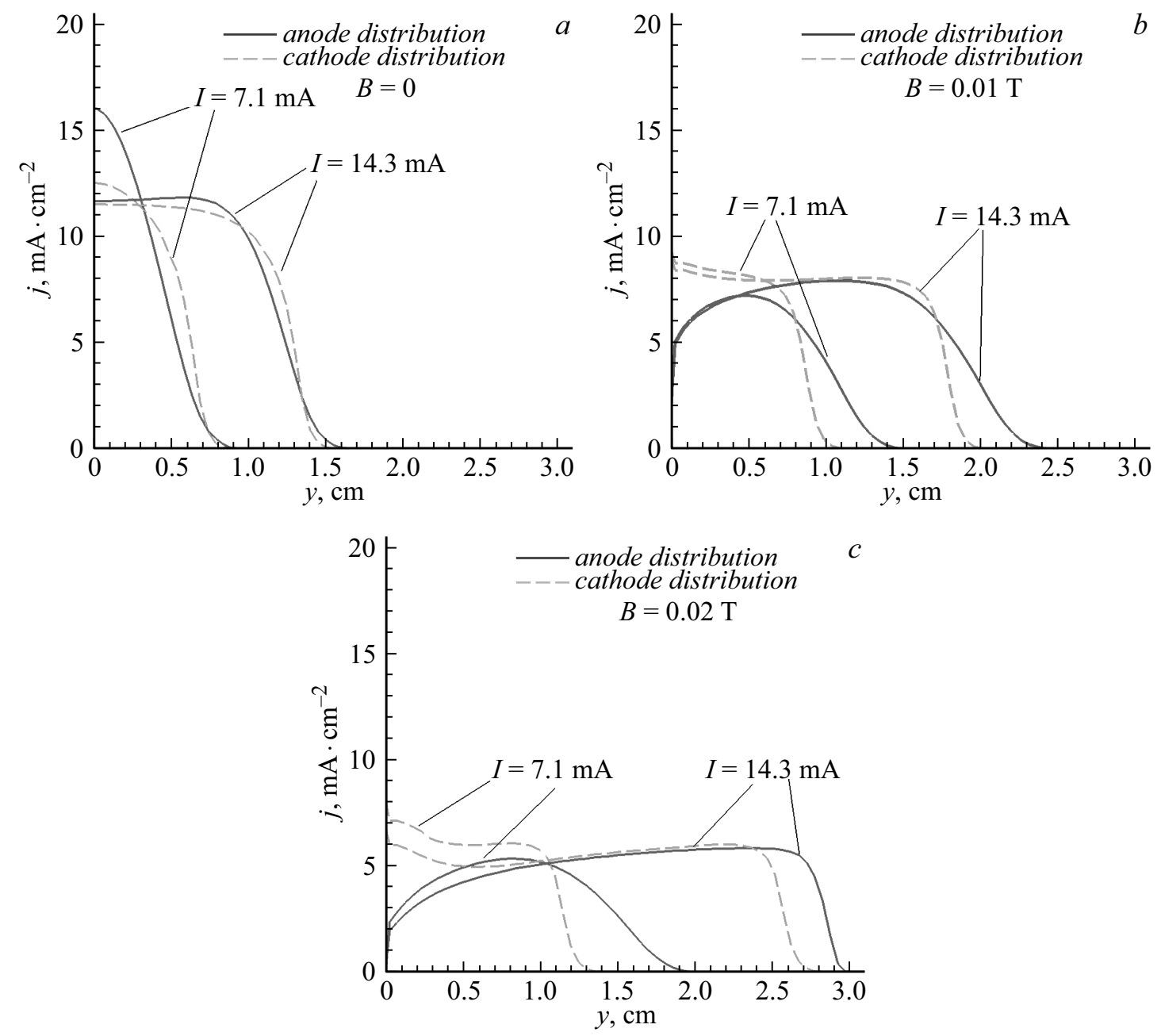

Рис. 4. Распределения плотностей тока на катоде и аноде в разрядах с различными магнитными полями для двух вариантов: $I=7.1$ и $14 \mathrm{~mA}$.

поля на электроны, проиллюстрирован на рис. 5. Наклон линии тока в области однородного положительного столба определяется отношением $\Gamma_{e y} / \Gamma_{e x} \approx \beta_{e}$, которое для рассматриваемого $B=0.02$ Т составляет $\beta_{e}=0.12$. Наклон линий, определенный из рисунка, лежит в пре-

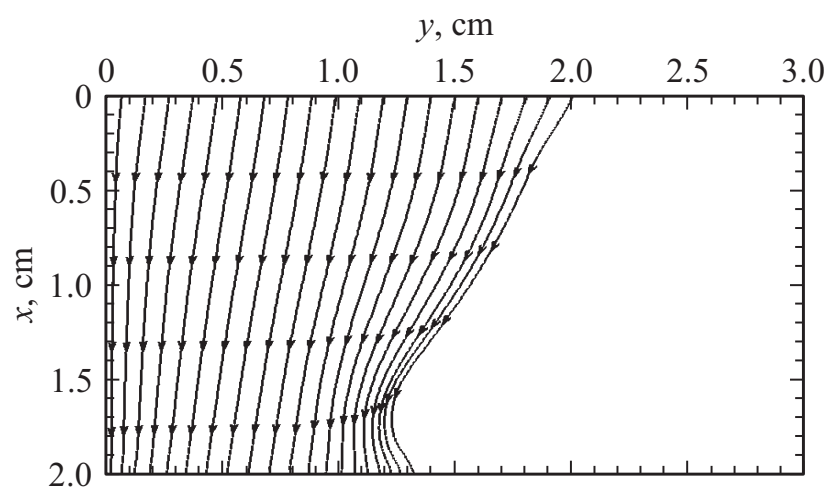

Рис. 5. Линии тока в разряде с магнитным полем $B=0.02 \mathrm{~T}$ для варианта рис. $2, c, I=7.1 \mathrm{~mA}$. Катод внизу. делах от 0.1 до 0.14, что согласуется с приведенной оценкой.

\section{Одномерная модель контракции положительного столба в магнитном поле}

В опытах [2,3] наблюдалось воздействие магнитного поля на контракцию разряда. Чем сильнее магнитное поле, тем до больших токов сохраняется диффузный разряд. Для изучения этого эффекта воспользуемся простейшей одномерной квазинейтральной моделью положительного столба, считая, что его параметры зависят только от поперечной току координаты $y$, а вдоль тока столб остается строго однородным. Те же допущения были положены в основу модели [3], в которой контракция в расчетах не проявилась, хотя в эксперименте она наблюдалась.

Разряд описываем системой уравнений квазинейтральной плазмы для плотности зарядов $n$ и газовой тем- 
пературы $T$. Уравнение для плотности $n$ получается стандартным образом $[5,6,12]$. Сначала приравниваем $y$-составляющие потоков электронов (4) и ионов (6) с учетом $D_{i}=0$, и из полученного равенства находим электрическое поле $E_{y}$. Затем подставляем найденное $E_{y}$ в любое из уравнений непрерывности (1) или (2), полагая $n_{e} \approx n_{i} \approx n$, и получаем уравнение амбиполярной диффузии плазмы в магнитном поле:

$$
\begin{gathered}
\frac{\partial n}{\partial t}+V_{H} \frac{\partial n}{\partial y}-\frac{\partial}{\partial y}\left(D_{a} \frac{\partial n}{\partial y}\right)=v_{\text {ion }} n-b_{\mathrm{eff}} n^{2}-v_{d} n \\
V_{H}=\mu_{+} \beta_{e} E_{x} \\
D_{a}=\mu_{+} T_{e} ; \quad v_{\text {ion }}=\alpha\left[E /\left(N \sqrt{1+\beta_{e}^{2}}\right)\right] \mu_{e \perp} E
\end{gathered}
$$

где поле $E \equiv E_{x}=$ const. Уравнение (11) уточняет выведенное в [3] слагаемыми с $D_{a}$ и $v_{d}$. Для газовой температуры $T$ пользуемся обычным уравнением теплопроводности:

$$
\begin{aligned}
N c_{p 1} \frac{\partial T}{\partial t}-\frac{\partial}{\partial y}\left(\lambda \frac{\partial T}{\partial y}\right) & =\eta_{T} j_{e} E+j_{i} E-v_{T}\left(T-T_{0}\right) \\
v_{T} & =\lambda \pi^{2} / h^{2}
\end{aligned}
$$

где $c_{p 1}-$ теплоемкость на одну молекулу, $\lambda-$ теплопроводность газа, $\eta_{T}$ - доля энерговыделения, которая идет в нагрев газа, $j_{e}$ и $j_{i}$ - электронный и ионный токи, $v_{T}$ - частота ухода тепла в плоскости щели. Уравнения (11) и (12) дополняются условием постоянства давления $p \sim N T=$ const и выражением для полного разрядного тока:

$$
I=E h \int_{0}^{L_{y}} \sigma(y) d y, \quad \sigma=e\left(\mu_{e \perp} n_{e}+\mu_{i \perp} n_{i}\right),
$$

где $\sigma-$ электропроводность. Граничные условия для (11)-(13) таковы: при $y=0, \partial n / \partial y=\partial T / \partial y=0$, при $y=L_{y}=15 \mathrm{~cm}, n=0, T=T_{0}=293 \mathrm{~K}$. Результаты расчетов несколько зависят от принятой доли энерговыделения $\eta_{T}$, которая идет в тепло. Мы рассматриваем эту величину как подгоночный параметр и принимаем $\eta_{T}=0.07$.

„Вольт-амперная“ характеристика $E=E(I)$ однородного вдоль $x$ разряда находилась путем решения уравнений (11)-(13) методом установления при разных токах $I$ и для магнитных полей $B=0,0.02,0.04 \mathrm{~T}$, как в эксперименте [3]. При слабых токах в качестве начальных условий задавались однородные распределения $n$ и $T$. После выхода разряда на стационарный режим мы увеличивали ток, а полученное стационарное решение использовалось в качестве начального условия.

Когда магнитное поле равно нулю, рассчитанная кривая $\mathrm{BAX} E=E(I)$ на рис. 6 монотонно падает и формально при любых токах соответствует контрагированному разряду с профилями $n, T$, спадающими как функции $y$ от $y=0$ (к обсуждению этого факта

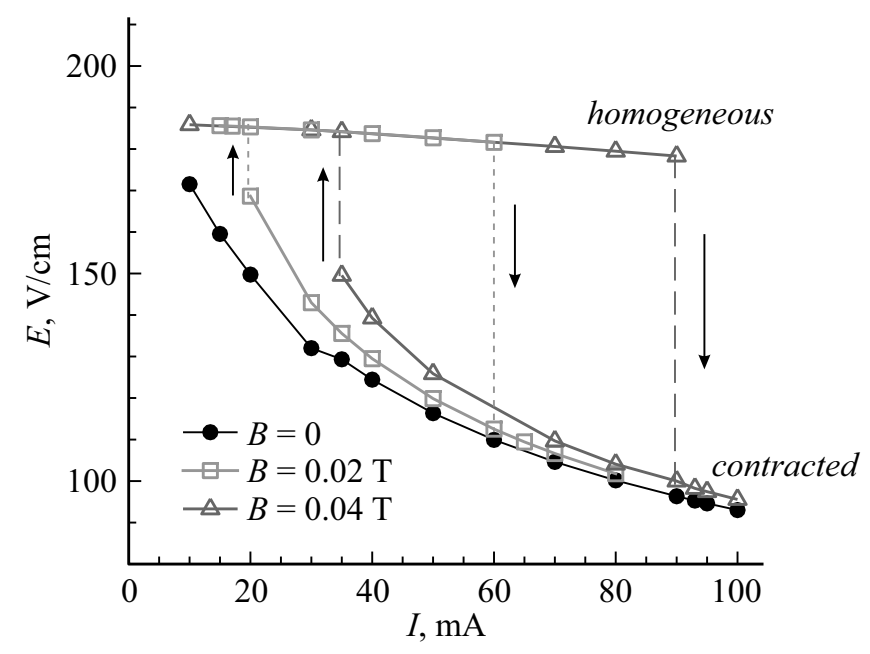

Рис. 6. „Вольт-амперная“ характеристика положительного столба разряда в азоте с магнитным полем, $p=15$ Torr, $L_{y}=15 \mathrm{~cm}$. Критические токи, при которых происходит скачкообразный переход от однородного состояния в контрагированное и обратно, показаны штриховыми линиями. Направления скачков на петлях гистерезиса показаны стрелками.

мы вернемся ниже). С увеличением тока распределения $n, T$ постепенно становятся более крутыми, степень заполнения током промежутка уменьшается, а плотность тока и температура при $y=0$ возрастают, т.е. эффект контракции становится более выраженным.

В магнитном поле разряд ведет себя иначе. При малых токах разряд пространственно однороден, а при увеличении тока скачком переходит в контрагированное состояние. Как видно из рис. 6, критический ток перехода к контракции растет с увеличением магнитного поля. Так, при $B=0.02 \mathrm{~T}, I_{\mathrm{cr}}=60 \mathrm{~mA}$, а при $B=0.04 \mathrm{~T}-$ $I_{\mathrm{cr}}=90 \mathrm{~mA}$. Переход между однородным и контрагированным состояниями имеет гистерезис.

Для исследования гистерезиса в качестве начального условия задается стационарное контрагированное состояние разряда, а ток постепенно уменьшается. Так рассчитывается нижняя ветвь „ВАХ“ на рис. 6 в разряде с магнитным полем. Когда ток становится меньше определенной величины $I_{1}$, разряд из контрагированного состояния переходит в однородное; $I_{1} \approx 20 \mathrm{~mA}$ при $B=0.02 \mathrm{~T} ; I_{1} \approx 35 \mathrm{~mA}$ при $B=0.04 \mathrm{~T}$ (рис. 6). Ширина петли гистерезиса увеличивается с ростом магнитного поля. При токах, соответствующих определенной петле гистерезиса, могут реализоваться как однородное, так и контрагированное состояния разряда. Выбор между ними определяется начальными условиями. Распределения $n$ и $T$ в обоих состояниях даны на рис. 7.

Хотя рассматриваемая модель и является грубым приближением к реальности в силу ее одномерности, рассчитанные „ВАХ“ можно качественно сравнить с вольт-амперными характеристиками разряда $V=V(I)$, измеренными в [3]. Как и в эксперименте, где при $B=0.02 \mathrm{~T} I_{\text {cr }}=65 \mathrm{~mA}$, при $B=0.04 \mathrm{~T} I_{\text {cr }}=105 \mathrm{~mA}$, в 

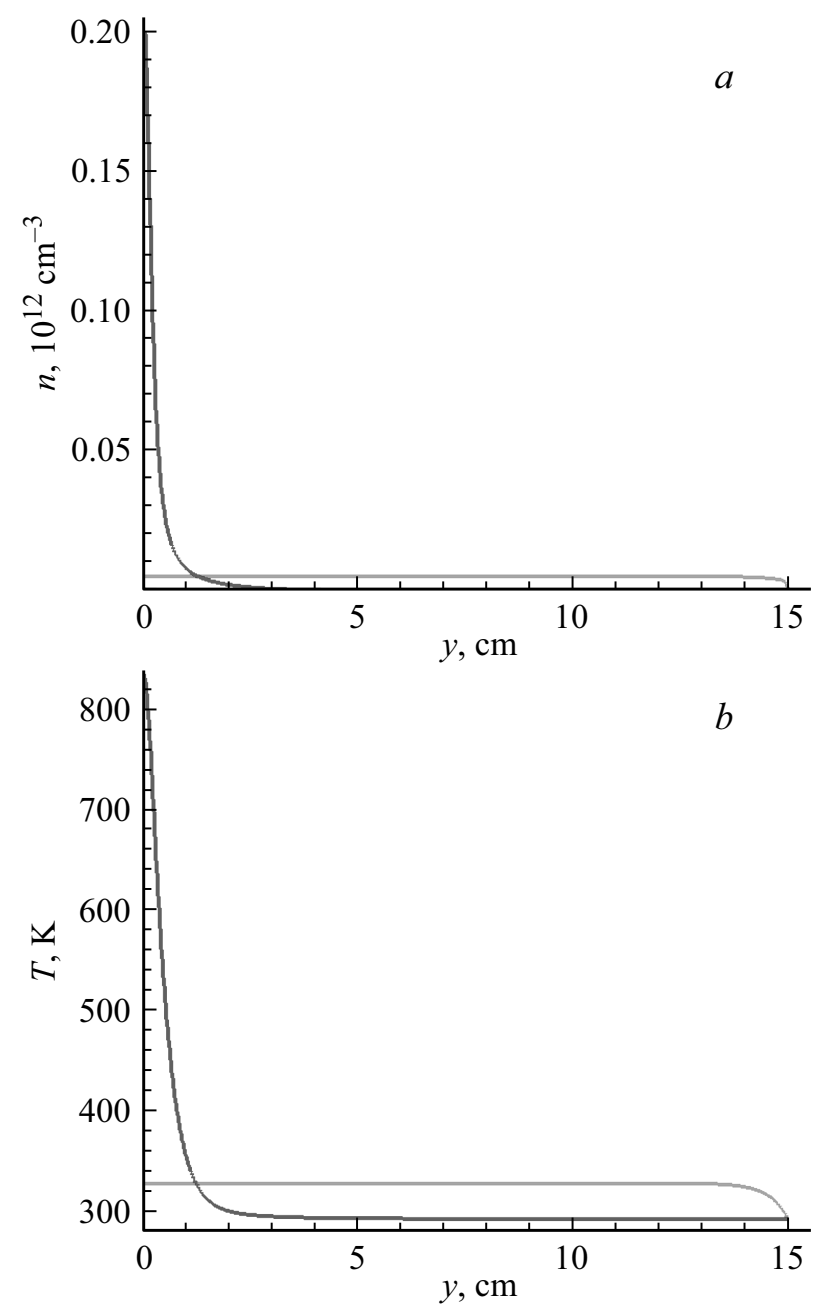

Рис. 7. Плотность плазмы $(a)$ и температура $(b)$ в однородном и контрагированном состояниях разряда при $I=80 \mathrm{~mA}$, $B=0.04 \mathrm{~T}$.

расчете критический ток перехода к контракции возрастает с ростом магнитного поля. В эксперименте в магнитном поле переход в контрагированное состояние происходил скачком, как и в нашей модели. В разряде без магнитного поля экспериментальная ВАX $V=V(I)$ спадает монотонно. Качественно подобное поведение мы наблюдаем и в расчете, где при $B=0$ скачка нет, но состояние заведомо контрагированное. Отсюда можно сделать предположение, что подобное поведение ВАХ на эксперименте [3] без магнитного поля свидетельствует о том, что разряд в эксперименте при примененных там токах тоже был контрагированным (насчет вида разряда без магнитного поля в [3] не сказано ничего, поэтому вопрос остается открытым).

Расчетное поле в однородном положительном столбе $E=180 \mathrm{~V} \cdot \mathrm{cm}^{-1}$ в магнитном поле хотя и в 1.8 раза выше экспериментального $E \approx\left(V-V_{\text {кс }}\right) / L_{x}=$ $=100 \mathrm{~V} \cdot \mathrm{cm}^{-1}$, оцененного по данным [3] $V=2.7 \mathrm{kV}$, $L_{x}=25 \mathrm{~cm}$ и рассчитанного нами падения напряжения на катодном слое $V_{\text {кс }} \approx 200 \mathrm{~V}$, но оно соответствует
$E / p=12 \mathrm{~V} \cdot \mathrm{cm}^{-1} \cdot \operatorname{Torr}^{-1}$, что не выходит за пределы теоретических значений приведенных полей в положительном столбе [12], которые получаются при той же принятой скорости ионизации.

Отметим, что вне зависимости от величины магнитного поля при малых токах рассматриваемая одномерная модель приводит к противоречию. В самом деле, в рамках этой модели сколь угодно малый ток автоматически окажется равномерно „размазанным“ по зазору площади $L_{y} \times h=15 \mathrm{~cm}^{2}$, так что плотность тока в столбе может оказаться в произвольное число раз меньше нормальной плотности тока на катоде, что нелепо. В реальности, как уже говорилось, источником тока для столба служит приэлектродный катодный слой. Поэтому ширина столба и плотность тока в нем определяются шириной катодного слоя, зависящей от тока, и геометрическим фактором - длиной разряда вдоль направления $x, L_{x}$. Для количественного исследования зависимости ширины столба от этих параметров с учетом возможной контракции разряда необходимо двумерное моделирование с реальными $L_{x}$ и $L_{y}$. Эта задача будет решаться нами в ближайшее время.

\section{Заключение}

Проведенное двумерное моделирование тлеющего разряда в диффузионно-дрейфовом приближении без учета нагрева газа позволило качественно продемонстрировать наблюдаемое в эксперименте заполнение разрядом плоского щелевого объема в поперечном магнитном поле. Чем сильнее магнитное поле, тем больше ширина разряда при фиксированном токе, тем меньше плотность тока на катоде и плотность плазмы в положительном столбе. При увеличении магнитного поля от нуля до $0.02 \mathrm{~T}$ плотность тока на катоде уменьшается вдвое.

В рамках приближения одномерного, бесконечно длинного положительного столба с учетом нагрева газа численно показано, что магнитное поле стабилизирует разряд, повышая критический ток перехода однородного состояния разряда в контрагированное. Этот результат находится в качественном согласии с экспериментом. Как показал расчет, при переходе от однородного состояния в контрагированное и обратно наблюдается значительный гистерезис.

Работа поддержана грантом РНФ 16-11-10275.

\section{Список литературы}

[1] Macken J. // Proc. Laser Advanced Materials Processing (LAMP'92). 1992. Vol. 1. P. 67-72.

[2] Яценко Н.А., Масюков И.В. // Письма в ЖТФ. 1993. Т. 19. Вып. 15. C. 17-21.

[3] Masyukov I.V., Myshenkov V.I. // J. Phys. D: Appl. Phys. 1994. Vol. 27. P. 2666-2669. DOI: $10.1088 / 0022-3727 / 27 / 12 / 032$ 
[4] Surzhikov S.T., Shang J.S. // J. Computational Physics. 2004. Vol. 199. P. 437-464. DOI: 10.1016/j.jcp.2004.02.019

[5] Суржсиков С.T. Физическая механика газовых разрядов. М.: Изд-во МГТУ им. Н.Э. Баумана, 2006. 640 с.

[6] Surzhikov S.T. Computational Physics of Electric Discharges in Gas Flows. Walter de Gruyter GmbH. Berlin/Boston. 2013. $428 \mathrm{p}$.

[7] Willis R.J., Seguin H.J.J., Capjack C.E., Nikumb S.K. // J. Applied Physics. 1987. Vol. 62. P. 3616-3620. DOI: $10.1063 / 1.339264$

[8] Ose E., Triebel W., Schumann A. // Contrib. Plasma Phys. 1994. Vol. 34. N 5. P. 649-657. DOI: $10.1002 /$ ctpp. 2150340504

[9] Голубев В.С., Кривенко Ю.Н., Леонов П.Г., Флеров В.Б. Письма в ЖТФ. 1988. Т. 14. Вып. 16. С. 1522-1526.

[10] Nath A.K., Chaubey R.S., RamKumar U.V.S., Chowdhary P., Kumar M., Abhinandan L. // IEEE J. Quantum Electronics. 1991. Vol. 27. N 3. P. 476-479. DOI: $10.1109 / 3.81350$

[11] Sohbatzadeh F., Tavassoli H., Latifi H. // Phys. Plasm. 2004. Vol. 11. P. 3904-3910. DOI: 10.1063/1.1767833

[12] Райзер Ю.П. Физика газового разряда. М.: Наука, Физматлит. 1987; 1992, 3-е изд., испр. и доп. Долгопрудный: Издательский дом „Интеллект“. 2009. 736 с. [Yu.P. Raizer, Gas Discharge Physics (Springer, Berlin, 1991, 1997), 449 p.]

[13] Tonks L. // Phys. Rev. 1937. Vol. 51. P. 744. DOI: 10.1103/PhysRev.51.744; Tonks L., Allis W.P. // Phys. Rev. 1937. Vol. 52. P. 710-713. DOI: 10.1103/PhysRev.52.710

[14] Райзер Ю.П., Суржсиков С.Т. // ТВТ. 1990. Т. 28. Вып. 3. C. 439-443. [Raizer Yu.P., Surzhikov S.T. // High Temperature, 1990. Vol. 28. N 3. P. 324-327.]

[15] Гинзбург В.Л., Гуревич А.В. // УФН. 1960. Т. 70. C. 201-246. DOI: 10.3367/UFNr.0070.196002a.0201. [Ginzburg V.L., Gurevich A.V. // Sov. Phys. Usp. 1960. Vol. 3. P. 115-146. DOI: 10.1070/PU1960v003n01ABEH003261.]

[16] Vahedi V., Dipeso G. // J. Computational Physics. 1997. Vol. 131. P. 149-163. DOI: 10.1006/jcph.1996.5591

[17] Райзер Ю.П., Суржиков С.Т. // ТВТ. 1988. Т. 26. Вып. 3. C. 428-435. [Raizer Yu.P, Surzhikov S.T. // High Temperature, 1988. Vol. 28. N 3. P. 304-311.] 